\title{
Adherence to COPD treatment in Turkey and Saudi Arabia: results of the ADCARE study
}

This article was published in the following Dove Press journal: International Journal of COPD

\author{
Nurdan Kokturk' \\ Mehmet Polatli \\ I Kivilcim Oguzulgen' \\ Sarfraz Saleemi ${ }^{3}$ \\ Mohammed Al Ghobain ${ }^{4}$ \\ Javed Khan ${ }^{5}$ \\ Adam Doble ${ }^{6}$ \\ Luqman Tariq ${ }^{7}$ \\ Fayaz Aziz ${ }^{7}$ \\ Abdelkader El Hasnaoui ${ }^{7}$ \\ 'Department of Pulmonary Medicine, \\ School of Medicine, Gazi University, \\ Besevler, Turkey; ${ }^{2}$ Department of \\ Pulmonary Medicine, School of \\ Medicine, Adnan Menderes University, \\ Aydin, Turkey; ${ }^{3}$ Section of Pulmonary \\ Medicine, Department of Medicine, \\ King Faisal Specialist Hospital and \\ Research Center, Riyadh, Saudi \\ Arabia; ${ }^{4}$ Department of Medicine, \\ College of Medicine, King Saud bin \\ Abdulaziz University for Health \\ Sciences, Riyadh, Saudi Arabia; \\ ${ }^{5}$ Department of Medicine, King Fahd \\ Armed Forces Hospital, Jeddah, Saudi \\ Arabia; ${ }^{6}$ Foxymed, Paris, France; \\ ${ }^{7}$ GlaxoSmithKline, Dubai, UAE
}

Background: COPD affects millions of people worldwide. Poor treatment adherence contributes to increased symptom severity, morbidity and mortality. This study was designed to investigate adherence to COPD treatment in Turkey and Saudi Arabia.

Methods: An observational, cross-sectional study in adult COPD patients in Turkey and Saudi Arabia. Through physician-led interviews, data were collected on sociodemographics and disease history, including the impact of COPD on health status using the COPD Assessment Test (CAT); quality of life, using the EuroQol Five-Dimension questionnaire (EQ-5D); and anxiety and depression using the Hospital Anxiety and Depression Scale (HADS). Treatment adherence was measured using the 8-item Morisky Medication Adherence Scale (MMAS-8). Multivariate logistic regression analysis examined the predictors of non-adherence and the impact of adherence on symptom severity.

Results: Four hundred and five COPD patients participated: 199 in Turkey and 206 in Saudi Arabia. Overall, 49.2\% reported low adherence (MMAS- $8<6$ ). Of those, $74.7 \%$ reported high disease impact (CAT $>15)$ compared to $58.4 \%$ reporting medium/high adherence $(p=0.0008)$. Patients with low adherence reported a lower mean 3-level EQ-5D utility value $(0.54 \pm 0.35)$ compared to those with medium/high adherence $(0.64 \pm 0.30 ; p<0.0001)$. Depression with HADS score $8-10$ or $>10$ was associated with lower adherence (OR 2.50 [95\% CI: 1.43-4.39] and 2.43 [95\% CI: 1.39-4.25], respectively; $p=0.0008)$. Being a high school/college graduate was associated with better adherence compared with no high school (OR 0.57 [95\% CI: 0.33-0.98] and 0.38 [95\% CI: 0.15-1.00], respectively; $p=0.0310$ ). After adjusting for age, gender, and country, a significant association between treatment adherence (MMAS- 8 score $\geq 6$ ) and lower disease impact (CAT $\leq 15)$ was observed (OR 0.56 [95\% CI: 0.33-0.95]; $p=0.0314$ ).

Conclusion: Adherence to COPD treatment is poor in Turkey and Saudi Arabia. Non-adherence to treatment is associated with higher disease impact and reduced quality of life. Depression, age, and level of education were independent determinants of adherence.

Keywords: COPD, non-adherence, 8-item Morisky Medication Adherence Scale, MMAS-8, EuroQol Five-Dimension questionnaire, EQ-5D, COPD Assessment Test, CAT, Turkey, Saudi Arabia

\section{Introduction}

COPD is a major contributor to chronic morbidity and mortality. It is estimated to have caused almost 3.2 million deaths globally in 2015 (equating to $\sim 6 \%$ of all deaths $)^{1}$ and is projected to be the third leading cause of death in middle-income countries by $2030 .^{2}$ COPD can be a debilitating illness, which has a huge impact on quality of life. It was estimated to be responsible for 63.8 million disability-adjusted life years in $2015 .^{3}$ Although not curable, it is treatable and symptom management is crucial.
Correspondence: Abdelkader El Hasnaoui GlaxoSmithKline, PO Box 50199,

Dubai, UAE

Tel +97I 44096305

Fax +97| 4332307 I

Email abdelkader.a.el-hasnaoui@gsk.com
International Journal of COPD 2018:13 1377-1388

(c) (1) (8) ( 2018 kokturk et al. This work is published and licensed by Dove Medical Press Limited. The full terms of this license are available at https://www.dovepress.com/terms.php

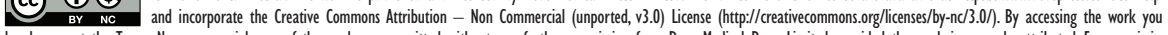
hereby accept the Terms. Non-commercial uses of the work are permitted without any further permision from Dove Medical Press Limited, provided the work is properly attributed. For permission for commercial use of this work, please see paragraphs 4.2 and 5 of our Terms (https://www.dovepress. con/terms.php). 
As with other chronic diseases, successful symptom management depends on many factors, one of which is patient adherence to the treatment regimen, yet studies suggest that many patients with chronic diseases do not use their medications as recommended. For example, a study carried out in Italy found that only $39.3 \%$ of outpatients with chronic conditions were adherent to their medication over the 4 weeks preceding their physician visit. ${ }^{4}$ A separate study in Saudi Arabia investigating medication use for chronic conditions reported that only $32.7 \%$ of medications were used exactly as prescribed. ${ }^{5}$ Reported adherence to COPD therapy specifically is poor. A recent study in Copenhagen reported the levels of adherence among COPD patients as ranging from $25 \%$ to $68 \%$ depending on the treatment regimen, ${ }^{6}$ and in a study in the USA, $58 \%$ of patients were found to be non-adherent to their COPD medications. ${ }^{7}$

There are limited studies that have examined adherence to COPD therapy specifically in the Middle East and Turkey. The available data come from small studies often carried out in a single hospital and the study designs are diverse. ${ }^{8-10}$ To address this unmet need, the ADCARE study was conducted. The primary objective of the study was to estimate the adherence to treatment in asthma and COPD in Turkey and Saudi Arabia under real-world care conditions using a standardized protocol. The secondary objectives were to collect data on disease characteristics, disease management, and health care resource utilization; to assess the main risk factors for nonadherence; and to analyze the relationship between adherence to treatment and asthma control/impact of COPD on health status. The results presented here will focus only on COPD.

\section{Methods}

\section{Study overview}

The ADCARE study is an observational, cross-sectional, multicenter study conducted in adult patients with either COPD or asthma in Turkey and Saudi Arabia. The study was conducted between September 2014 and August 2016.

A steering committee of experts specializing in COPD and asthma, in addition to appropriate representatives from the sponsor project team, was established to provide input on the study design and the scientific operation of the study, to review the data analyses, and to provide recommendations on publication strategy. Data were collected by contract research organizations (CROs) in Turkey (ZeinCRO) and Saudi Arabia (Clinart). Data management and analysis was performed by MS Health (Morocco).

\section{Feasibility study}

Prior to initiating the main study, a feasibility study was performed in each participating country in order to investigate the most appropriate method for recruiting patients and to assess the acceptability of the case report form (CRF) and the method of data collection.

\section{Recruitment of investigators}

In each country, specialists in respiratory disease (pulmonologists, internists, or other respiratory/allergy specialists) were approached for participation in the study. Physicians were randomly selected from established hospitals treating COPD patients. The eligible physicians had to have a track record in conducting observational studies and have awareness of Good Clinical Practice (GCP), in addition to the availability of structured patient records. Physicians were recruited from multiple regions across the 2 countries. In Saudi Arabia, physicians from the Central, Western, Southern, and Eastern regions participated. In Turkey, physicians from the Central Anatolia, Aegean, Marmara, Mediterranean, Black Sea, and South Eastern Anatolia regions participated. Ensuring physician representation across several regions of the participating countries enabled a representative patient sample from across the country to be recruited.

At first contact, a short overview of the study was provided to the physician, and written agreement to participate as an investigator was obtained. All physicians were trained in correct CRF completion and instructions for patient-reported outcomes (PROs) and received training on reporting adverse events, prior to patient enrolment at the site.

\section{Recruitment of patients}

A sample size of 200 COPD patients was targeted from each country in order to estimate the adherence with a precision $(95 \% \mathrm{CI})$ of $\pm 7.5 \%$ and a statistical power $(1-\beta)$ of $80 \%$. The total number of COPD patients targeted to take part in the study was 400 .

During a 3-month inclusion period, participating investigators recruited all successive patients attending a spontaneous or previously planned consultation with their doctors and who fulfilled the eligibility criteria, until the prespecified target of 400 patients was reached. All subjects who 1) were aged $\geq 40 ; 2$ ) had a documented COPD diagnosis $\geq 1$ year; 3 ) had been prescribed at least 1 COPD maintenance medication by one of the participating investigators in the 3 months prior to inclusion (including but not limited to inhaled corticosteroids [ICSs], long-acting beta agonist [LABA], long-acting muscarinic antagonist [LAMA], ICS/LABA, ICS+LAMA, ICS/LABA+LAMA, and/or phosphodiesterase type 4 inhibitors or any combination of these); 4) were informed of the study objectives and provided their written consent, were 
eligible unless the prespecified quota had already been filled. If the subject was not eligible, this was documented. Reasons for ineligibility were severe psychiatric illness or other disease that could compromise participation in the study or current participation in a clinical trial or cohort study.

\section{Data collection by the investigating physician}

During the patient visit, once written informed consent to participate was obtained, data were collected on sociodemographics (such as age, gender, weight, education level, and smoking status), comorbidities, the patient's disease history related to COPD, disease management, and COPDrelated health care resource utilization. Some of these data were ascertained retrospectively from the patient's medical records.

\section{PROs}

The impact of COPD on health status was assessed using the 8-item COPD Assessment Test (CAT). ${ }^{11}$ In this study, a CAT score of $<10$ equates to low impact, 10-15 signifies medium impact, and $>15$ denotes high impact. The level of breathlessness experienced by the patient was assessed via the Medical Research Council (MRC) dyspnea scale; ${ }^{12}$ this is scored on a single scale from 1 ("I only get breathless with strenuous exercise") to 5 ("I'm too breathless to leave the house"). Quality of life was assessed using the 3-level EuroQol Five-Dimension questionnaire (EQ-5D-3L) - a self-report tool comprising a 5-item health status measure from which a utility value is calculated and a EuroQol Visual Analog rating Scale (EQ-VAS) (c) EuroQol Research Foundation. EQ-5D ${ }^{\mathrm{TM}}$ is a trade mark of the EuroQol Research Foundation). ${ }^{13,57}$ On the advice of the EuroQol Research Foundation, the EQ-5D-3L utility values presented were calculated based on the UK value sets, in the absence of country-specific data for Turkey and Saudi Arabia. The Hospital Anxiety and Depression Scale (HADS) was used to assess the levels of anxiety and depression experienced by the patient. ${ }^{14}$ A score of $<8$ represented a patient who was not anxious/depressed; a score between 8 and 10 represented a patient who was questionably anxious/depressed; and a score $>10$ indicated a patient who was anxious/depressed.

Adherence to treatment was measured by the 8-item Morisky Medication Adherence Scale (MMAS-8), an 8-item self-report questionnaire which has been validated and used widely in many disease areas. ${ }^{15-17}$ Question 1 asks, "Do you sometimes forget to take your medication?"; question 2 asks, "People sometimes miss taking their medications for reasons other than forgetting. Thinking over the past 2 weeks, were there any days when you did not take your medication?"; question 3 asks, "Have you ever cut back or stopped taking your medication without telling your doctor, because you felt worse when you took it?"; question 4 asks, "When you travel or leave home, do you sometimes forget to bring along your medication?"; question 5 asks, "Did you take your medication yesterday?"; question 6 asks, "When you feel like your condition is under control, do you sometimes stop taking your medication?"; question 7 asks, "Taking medication every day is a real inconvenience for some people. Do you ever feel hassled about sticking to your treatment plan?"; question 8 asks, "How often do you have difficulty remembering to take all your medications?". The responses for the items are yes/no except for the last item, which is scored on a 5-point Likert scale. The score was calculated according to the author's instructions, and the possible score ranges from 0.0 to 8.0. A score of $<6.0$ indicated low adherence to treatment, while scores between 6.0 and $<8.0$, and 8.0 were categorized as medium and high adherence, respectively. ${ }^{17}$ Written permission was obtained from the copyright owners of the MMAS-8 questionnaire for any excerpts from copyrighted works that are included and the sources have been credited in the article.

Validated, local language versions (Arabic and Turkish) of the questionnaires were used in each country. In Saudi Arabia, a proportion of questionnaires were completed in English at the patient's request.

\section{Statistical analysis}

Categorical and ordinal variables are presented as frequency and counts. Continuous variables are presented as mean values with standard deviation. Univariate and multivariate analysis were used to identify the principal risk factors associated with poor adherence. Potential associations were tested using the $\chi^{2}$-test, Fisher's exact test, or the Kruskal-Wallis test, as appropriate. The variables reported on the physician and patient questionnaires were assessed for association with non-adherence. In the first step, each variable was evaluated independently in a univariate analysis. All variables with a $p$-value of $<0.20^{18}$ in the univariate analysis were entered into the multiple logistic regression analysis in which variables were retained in the model using a backward selection in order to determine the variables that were associated with non-adherence at the probability level of 0.05 . A final multivariate analysis was conducted to generate ORs. The relationship between the impact of COPD on health status and adherence to treatment was also assessed via multiple logistic regression analysis adjusting for age, gender, and 
country as potential confounding factors. Two-sided tests were used throughout and a probability level of 0.05 considered as statistically significant unless otherwise specified. Analyses were performed using SAS 9.4 (SAS Institute Inc., Cary, NC, USA).

\section{Ethics approval}

This study was conducted in accordance with the guidelines for good pharmacoepidemiology practices and ICH GCP as this applies to observational research. In Turkey, the ADCARE study was approved by Gazi University Ethics Committee (Gazi Üniversitesi Klinik Araştırmalar Etik Kurulu), in line with the country practice for multicentered studies where a single ethics committee approval obtained from the coordinator site's ethics committee is sufficient. In Saudi Arabia, the study was approved by the following ethics committees: Institutional Review Board, King Abdullah International Medical Research Center; Clinical Research Committee and Research Ethics Committee at King Faisal Specialist Hospital; Institutional Review Board of the Central Region; King Fahad Medical City Institutional Review Board; King Khaled University Hospital Institutional Review Board; Air Base Dammam Research and Ethics Committee; Research Ethics Committee, Unit of Biomedical Ethics-King Abdelaziz University; Institutional Review Board at King Fahd Military Hospital; Research Ethics Committee in King Khalid University; and King Fahad Medical City Institutional Review Board, Medina. Subjects provided written informed consent to participate in the study. All data collected were kept confidential and anonymous. Participating subjects did not receive any financial compensation for their participation in the study.

\section{Results}

\section{Study recruitment}

In Saudi Arabia, a total of 22 physicians were invited to participate. Of these, 8 refused to take part, 2 did not respond, 1 did not sign the study contract, and 1 dropped out. Therefore, 10 physicians participated in the study as investigators, who recruited a mean number of 17 COPD patients per site.

In Turkey, a total of 35 physicians were invited to participate. Four sites were not initiated or dropped out. Therefore, 31 physicians participated in the study as investigators, who recruited a mean number of 6 COPD patients per site.

\section{Study population}

A total of 405 patients with COPD agreed to participate in the study. This population was evenly distributed between
Turkey $(n=199)$ and Saudi Arabia $(n=206)$. Demographics of the overall study population and the individual countries studied are shown in Table 1 . The majority of respondents were men $(81.5 \% ; n=330),>60$ years of age $(72.1 \% ; n=292)$, either past or current smokers $(80.7 \% ; n=327)$, overweight or obese $(62.5 \% ; n=248)$, and reported at least 1 comorbidity $(70.6 \% ; n=286)$.

\section{Disease characteristics}

Overall, the mean age at symptom onset was 52.3 years of age and the mean disease duration was 10 years. Approximately

Table I Demographics of the study population

\begin{tabular}{|c|c|c|c|}
\hline Variable & $\begin{array}{l}\text { Saudi Arabia } \\
n=206\end{array}$ & $\begin{array}{l}\text { Turkey } \\
n=199\end{array}$ & $\begin{array}{l}\text { Overall } \\
n=405\end{array}$ \\
\hline \multicolumn{4}{|l|}{ Gender, n (\%) } \\
\hline Count & 206 & 199 & 405 \\
\hline Men & $155(75.2)$ & $175(87.9)$ & $330(8 \mid .5)$ \\
\hline Women & $51(24.8)$ & $24(12.1)$ & $75(18.5)$ \\
\hline \multicolumn{4}{|l|}{ Age, n (\%) } \\
\hline Count & 206 & 199 & 405 \\
\hline $40-49$ years & $6(2.9)$ & $15(7.5)$ & $21(5.2)$ \\
\hline $50-59$ years & $42(20.4)$ & $50(25.1)$ & $92(22.7)$ \\
\hline$\geq 60$ years & $158(76.7)$ & $134(67.3)$ & $292(72.1)$ \\
\hline \multicolumn{4}{|l|}{ Body mass index, n (\%) } \\
\hline Count & 200 & 197 & 397 \\
\hline Underweight & $2(1.0)$ & II (5.6) & $13(3.3)$ \\
\hline Normal weight & $58(29.0)$ & $78(39.6)$ & $136(34.3)$ \\
\hline Overweight & $66(33.0)$ & $64(32.5)$ & $130(32.8)$ \\
\hline Obese & $74(37.0)$ & $44(22.3)$ & II 8 (29.7) \\
\hline \multicolumn{4}{|l|}{ Educational level, n (\%) } \\
\hline Count & 200 & 183 & 383 \\
\hline No high school & $146(73.0)$ & $115(62.8)$ & $26 \mid(68.2)$ \\
\hline High school graduate & $43(21.5)$ & $45(24.6)$ & $88(23.0)$ \\
\hline College graduate & II (5.5) & $23(12.6)$ & $34(8.9)$ \\
\hline \multicolumn{4}{|l|}{ Health system coverage, $n(\%)$} \\
\hline Count & 198 & 198 & 396 \\
\hline Social security/public & $182(91.9)$ & I88 (95.0) & $370(93.4)$ \\
\hline Private/insured & $10(5.1)$ & $2(1.0)$ & $12(3.0)$ \\
\hline Not insured & $6(3.0)$ & I (0.5) & $7(1.8)$ \\
\hline Other & 0 & $7(3.5)$ & $7(1.8)$ \\
\hline \multicolumn{4}{|l|}{ Smoking status, n (\%) } \\
\hline Count & 206 & 199 & 405 \\
\hline Non-smoker & $67(32.5)$ & II (5.5) & $78(19.3)$ \\
\hline Past-smoker & $97(47.1)$ & $144(72.4)$ & $24 \mid(59.5)$ \\
\hline Smoker & $42(20.4)$ & $44(22.1)$ & $86(21.2)$ \\
\hline \multicolumn{4}{|l|}{ Pack-years, n (\%) } \\
\hline Count & 130 & 166 & 296 \\
\hline$<10$ pack-years & $5(3.9)$ & $5(3.0)$ & $10(3.4)$ \\
\hline$\geq 10$ pack-years & $125(96.2)$ & 161 (97.0) & $286(96.6)$ \\
\hline \multicolumn{4}{|c|}{ Number of comorbidities, n (\%) } \\
\hline Count & 206 & 199 & 405 \\
\hline No comorbidity & $40(19.4)$ & 79 (39.7) & $119(29.4)$ \\
\hline At least one comorbidity & $166(80.6)$ & $120(60.3)$ & $286(70.6)$ \\
\hline
\end{tabular}

Note: Sociodemographics of the study population: distribution of patients, by age, gender, country, body mass index, health system coverage, level of education, smoking status, and presence of comorbidities. 
half $(52.5 \% ; n=209)$ of all patients reported having had an exacerbation in the last 12 months, and 43.7\% ( $n=164)$ reported having used oxygen therapy in the last 6 months. In addition, $66.6 \%(n=261)$ of patients reported high disease impact (CAT $>15)$, and the vast majority of patients $(91.1 \%$; $\mathrm{n}=356$ ) reported an MRC score of $>1$.

In Saudi Arabia, patients appear to present with more severe disease compared with Turkey. A higher percentage of patients (61.3\%; $n=122)$ in Saudi Arabia reported having had an exacerbation in the last 12 months compared with Turkey (43.7\%; $\mathrm{n}=87 ; p=0.0004)$, and a higher percentage of patients reported a CAT score of $>15$ in Saudi Arabia $(77.2 \%$; $=152)$ compared with Turkey $(55.9 \% ; \mathrm{n}=109 ; p<0.0001)$.

The overall EQ-5D-3L utility score reported by the patients was 0.59, and the EQ-VAS score was 65.2. Less than half of the overall study population were classed as anxious $(42.9 \% ; n=168)$ and approximately half were classed as depressed $(54.1 \%$; $=211$; HADS anxiety or depression score $\geq 8$ ). These results are presented in Table 2 .

\section{Disease management}

Overall, the majority of patients $(68.9 \% ; n=270)$ had undergone a lung function test in the last 6 months. Of those who did undergo the examination, $58.3 \%(n=151)$ had a last value of forced expiratory volume in 1 second $\left(\mathrm{FEV}_{1}\right)>50 \%$, and $48.1 \%(n=124)$ had a last value of forced vital capacity (FVC) $>70 \%$.

At a country level, almost all patients had undergone a lung function test in Turkey $(91.8 \% ; n=178)$ compared with less than half of the patients in Saudi Arabia (46.5\%; $n=92$; $p<0.0001$ ). In addition, only $52 \%$ of patients in Turkey who had a lung function test in the last 6 months had an $\mathrm{FEV}_{1}$ value of $>50 \%$ compared to $71.4 \%$ of those in Saudi Arabia $(p=0.0041)$. These results are presented in Table 3 .

\section{Adherence to COPD treatment}

Overall, 49.2\% $(n=190)$ of patients reported low adherence to treatment (MMAS-8 <6) and the mean Morisky score was 5.4. The mean MMAS-8 score was significantly higher in Turkey (6.2) compared with Saudi Arabia (4.6; $p<0.0001)$. A higher proportion of patients reported low treatment adherence in Saudi Arabia (64.2\%; $\mathrm{n}=122)$ compared with Turkey $(34.7 \% ; \mathrm{n}=68 ; p<0.0001)$. Overall, only $20 \%(n=77)$ of patients reported high treatment adherence (MMAS-8=8), and the proportion of highly adherent patients in Turkey $(28.1 \%$; $=55)$ was more than double that of Saudi Arabia (11.6\%; $n=22)$. These results are presented in Table 4.
Table 2 Disease characteristics

\begin{tabular}{|c|c|c|c|}
\hline Variable & $\begin{array}{l}\text { Saudi Arabia } \\
\mathrm{n}=\mathbf{2 0 6}\end{array}$ & $\begin{array}{l}\text { Turkey } \\
\mathrm{n}=199\end{array}$ & $\begin{array}{l}\text { Overall } \\
n=405\end{array}$ \\
\hline \multicolumn{4}{|c|}{ Age at symptom onset (years) } \\
\hline Count & 201 & 199 & 400 \\
\hline Mean $( \pm S D)$ & $51.6( \pm 12.1)$ & $52.9( \pm 10.9)$ & $52.3( \pm 11.5)$ \\
\hline \multicolumn{4}{|c|}{ Disease duration (years) } \\
\hline Count & 202 & 199 & 401 \\
\hline Mean $( \pm S D)$ & $11.7( \pm 10.0)$ & $8.3( \pm 7.5)$ & $10.0( \pm 9.0)$ \\
\hline \multicolumn{4}{|c|}{ Time between symptom onset and diagnosis (years) } \\
\hline Count & 199 & 196 & 395 \\
\hline Mean $( \pm S D)$ & $3.4( \pm 5.5)$ & $2.4( \pm 5.9)$ & $2.9( \pm 5.7)$ \\
\hline \multicolumn{4}{|c|}{ Exacerbations in the last 12 months, $n$ (\%) } \\
\hline Count & 199 & 199 & 398 \\
\hline No & 77 (38.7) & $112(56.3)$ & $189(47.5)$ \\
\hline Yes & $122(61.3)$ & $87(43.7)$ & $209(52.5)$ \\
\hline \multicolumn{4}{|c|}{ CAT score, $n(\%)$} \\
\hline Count & 197 & 195 & 392 \\
\hline$<10$ & $13(6.6)$ & $4 \mid(2 I .0)$ & $54(13.8)$ \\
\hline $10-15$ & $32(16.2)$ & $45(23.1)$ & $77(19.6)$ \\
\hline$>15$ & $152(77.2)$ & $109(55.9)$ & $261(66.6)$ \\
\hline \multicolumn{4}{|c|}{ MRC dyspnea scale, n (\%) } \\
\hline Count & 194 & 197 & 391 \\
\hline$M R C=1$ & $13(6.7)$ & $22(11.2)$ & $35(9.0)$ \\
\hline$M R C>I$ & I8I (93.3) & $175(88.8)$ & $356(91.1)$ \\
\hline \multicolumn{4}{|c|}{ EQ-5D-3L utility values } \\
\hline Count & 197 & 199 & 396 \\
\hline Mean $( \pm S D)$ & $0.52( \pm 0.37)$ & $0.66( \pm 0.27)$ & $0.59( \pm 0.33)$ \\
\hline \multicolumn{4}{|l|}{ EQ-VAS score } \\
\hline Count & 205 & 198 & 403 \\
\hline Mean $( \pm S D)$ & $70.3( \pm I 4.8)$ & $59.9( \pm 18.7)$ & $65.2( \pm 17.6)$ \\
\hline \multicolumn{4}{|c|}{ HADS anxiety score, $n(\%)$} \\
\hline Count & 195 & 197 & 392 \\
\hline$<8$ & $96(49.2)$ & $128(65.0)$ & $224(57.1)$ \\
\hline $8-10$ & $61(31.3)$ & $35(17.8)$ & $96(24.5)$ \\
\hline$>10$ & $38(19.5)$ & $34(17.3)$ & $72(18.4)$ \\
\hline \multicolumn{4}{|c|}{ HADS depression score, $n$ (\%) } \\
\hline Count & 193 & 197 & 390 \\
\hline$<8$ & $70(36.3)$ & $109(55.3)$ & $179(45.9)$ \\
\hline $8-10$ & $51(26.4)$ & $5 I(25.9)$ & $102(26.2)$ \\
\hline$>10$ & $72(37.3)$ & $37(18.8)$ & $109(28.0)$ \\
\hline
\end{tabular}

Notes: Disease characteristics of the study population. An exacerbation was defined as a hospitalization or emergency room visit.

Abbreviations: CAT, COPD Assessment Test; EQ-5D-3L, 3-level EuroQol FiveDimension questionnaire; EQ-VAS, EuroQol Visual Analog Scale; HADS, Hospital Anxiety and Depression Scale; MRC, Medical Research Council.

Overall, $74.7 \%(n=139)$ of patients who reported low adherence to treatment had a CAT score of $>15$, compared with only $58.4 \%(n=111)$ of those who reported medium/high treatment adherence ( $p=0.0008)$. In addition, those patients who reported low treatment adherence reported a lower EQ-5D-3L utility value $(0.54)$ compared with those with medium/high adherence $(0.64 ; p<0.0001)$. There was no significant difference observed in reported EQ-VAS scores between adherence classes. These data are presented in Table 5. 
Table 3 Disease management

\begin{tabular}{|c|c|c|c|}
\hline Variable & $\begin{array}{l}\text { Saudi Arabia } \\
n=206\end{array}$ & $\begin{array}{l}\text { Turkey } \\
\mathrm{n}=199\end{array}$ & $\begin{array}{l}\text { Overall } \\
n=405\end{array}$ \\
\hline \multicolumn{4}{|c|}{ Lung function test in the last 6 months, $\mathrm{n}(\%)$} \\
\hline Count & 198 & 194 & 392 \\
\hline No & $106(53.5)$ & $16(8.3)$ & $122(3||)$. \\
\hline Yes & $92(46.5)$ & $178(91.8)$ & $270(68.9)$ \\
\hline \multicolumn{4}{|l|}{ Last value of $\mathrm{FEV}_{1}, \mathrm{n}(\%)$} \\
\hline Count & 84 & 175 & 259 \\
\hline$<30 \%$ & $2(2.4)$ & $20(11.4)$ & $22(8.5)$ \\
\hline $30 \%-50 \%$ & $22(26.2)$ & $64(36.6)$ & $86(33.2)$ \\
\hline$>50 \%$ & $60(71.4)$ & $91(52.0)$ & $|5|(58.3)$ \\
\hline \multicolumn{4}{|l|}{ Last value of FVC, $n(\%)$} \\
\hline Count & 84 & 174 & 258 \\
\hline$<50 \%$ & II (I3.I) & $29(16.7)$ & $40(15.5)$ \\
\hline $50 \%-70 \%$ & $30(35.7)$ & $64(36.8)$ & $94(36.4)$ \\
\hline$>70 \%$ & $43(5 \mid .2)$ & $81(46.6)$ & $124(48.1)$ \\
\hline \multicolumn{4}{|c|}{ Oxygen therapy use in the last 6 months, $n(\%)$} \\
\hline Count & 195 & 180 & 375 \\
\hline No & $98(50.3)$ & $113(62.8)$ & $211(56.3)$ \\
\hline Yes & $97(49.7)$ & $67(37.2)$ & $164(43.7)$ \\
\hline \multicolumn{4}{|l|}{ Treatment class, $\mathrm{n}(\%)$} \\
\hline Count & 206 & 199 & 405 \\
\hline ICS/LABA/LAMA & $156(75.7)$ & I5I (75.9) & $307(75.8)$ \\
\hline ICS/LABA or ICS/LAMA & $36(17.5)$ & $34(17.1)$ & $70(17.3)$ \\
\hline LAMA or LABA & $8(3.9)$ & $10(5.0)$ & $18(4.4)$ \\
\hline SAMA or SABA & $5(2.4)$ & $3(1.5)$ & $8(2.0)$ \\
\hline Other & I (0.5) & I (0.5) & $2(0.5)$ \\
\hline \multicolumn{4}{|c|}{ Number of maintenance treatments (\%) } \\
\hline Count & 206 & 199 & 405 \\
\hline One treatment & $25(12.1)$ & $19(9.6)$ & $44(10.9)$ \\
\hline Two treatments & 81 (39.3) & $87(43.7)$ & $168(4 \mid .5)$ \\
\hline More than two treatments & $100(48.5)$ & $93(46.7)$ & $193(47.7)$ \\
\hline Mean $( \pm S D)$ & $2.6( \pm I . I)$ & $2.6( \pm 1.0)$ & $2.6( \pm I . I)$ \\
\hline \multicolumn{4}{|c|}{ Frequency of maintenance treatment, $n(\%)$} \\
\hline Count & 205 & 199 & 404 \\
\hline Once a day or less & $9(4.4)$ & $7(3.5)$ & $16(4.0)$ \\
\hline Twice a day & $178(86.8)$ & $135(67.8)$ & $313(77.5)$ \\
\hline More than twice a day & $18(8.8)$ & $57(28.6)$ & $75(18.6)$ \\
\hline \multicolumn{4}{|l|}{ Type of inhalation device, $\mathrm{n}(\%)$} \\
\hline Metered-dose inhaler & $51(24.8)$ & $64(32.2)$ & II 5 (28.4) \\
\hline Dry powder inhaler & $177(85.9)$ & $183(92.0)$ & $360(88.9)$ \\
\hline Nebulizer & $22(10.7)$ & $27(13.6)$ & $49(12.1)$ \\
\hline Other & 71 (34.5) & $5 I(25.6)$ & $122(30.1)$ \\
\hline
\end{tabular}

Notes: Disease management characteristics of the study population. The treatment class "other" includes ICS alone, and ICS and methylxanthine. The type of inhalation device "other" includes capsule, diskus, handihaler, inhaler capsule, injection, mask, nasal, nasal pillow, oral, spray, subcutaneous, subcutaneous injection, subcutanous, tablet, turbuhaler, vaccine, and nasal spray.

Abbreviations: $\mathrm{FEV}_{1}$, forced expiratory volume in I second; FVC, forced vital capacity; ICS, inhaled corticosteroid; LABA, long-acting beta agonist; LAMA, longacting muscarinic antagonist; SABA, short-acting beta agonist; SAMA, short-acting muscarinic antagonist.

\section{Predictors of non-adherence}

The univariate analysis showed that the following variables were found to have a significant $(p<0.20)$ association with low treatment adherence (defined as an MMAS-8 score of $<6$ ): gender, country, age, educational level, the presence
Table 4 Adherence to treatment

\begin{tabular}{|c|c|c|c|c|}
\hline Variable & $\begin{array}{l}\text { Saudi Arabia } \\
\mathrm{n}=\mathbf{2 0 6}\end{array}$ & $\begin{array}{l}\text { Turkey } \\
n=199\end{array}$ & $\begin{array}{l}\text { Overall } \\
n=405\end{array}$ & $p$-value \\
\hline \multicolumn{5}{|l|}{ Morisky score*, n (\%) } \\
\hline Count & 190 & 196 & 405 & \\
\hline Mean $( \pm S D)$ & $4.6( \pm 2.4)$ & $6.2( \pm \mathrm{I} .8)$ & $5.4( \pm 2.3)$ & $<0.0001$ \\
\hline$<6$ (low) & $122(64.2)$ & $68(34.7)$ & $190(49.2)$ & $<0.0001$ \\
\hline 6 to $<8$ (medium) & $46(24.2)$ & $73(37.2)$ & $119(30.8)$ & \\
\hline$=8$ (high) & $22(11.6)$ & $55(28.1)$ & $77(20.0)$ & \\
\hline
\end{tabular}

Notes: Adherence to treatment for the overall study population and by country, measured using the MMAS-8. The $p$-values were calculated using the $\chi^{2}$-test for the MMAS- 8 categories and the Kruskal-Wallis test for the MMAS- 8 mean score. *The MMAS (8-item) content, names, and trademarks are protected by the US copyright and trademark laws. Permission for use of the scale and its coding is required. A license agreement is available from Donald E Morisky, ScD, ScM, MSPH, I 4725 NE 20th St Bellevue, WA 98007, USA; dmorisky@gmail.com.

Abbreviation: MMAS-8, 8-Item Morisky Medication Adherence Survey.

of comorbidities, anxiety, depression, MRC score, the number of exacerbations, and last value of $\mathrm{FEV}_{1}$.

More detailed investigation using a backward step-wise multivariate regression analysis revealed that, of those variables, living in Saudi Arabia was associated with lower treatment adherence (OR 3.20; $p=0.0001$ ) compared with Turkey. In addition, HADS depression $8-10$ or $>10$ was also associated with lower treatment adherence (OR 2.50 and 2.43 , respectively; $p=0.0008$ ). Higher education (high school or college graduate) was associated with better treatment adherence compared to not graduating high school (OR 0.57 and 0.38 , respectively; $p=0.0310$ ), and being $\geq 60$ years

Table 5 Association between adherence to treatment, impact of COPD on health status, and quality of life

\begin{tabular}{|c|c|c|c|c|}
\hline ariable & & $\begin{array}{l}\text { Low adherence } \\
\text { (MMAS-8 <6) } \\
n=190\end{array}$ & $\begin{array}{l}\text { Medium } \\
\text { or high } \\
\text { adherence } \\
\text { (MMAS-8 } \geq 6 \text { ) } \\
n=196\end{array}$ & $p$-value \\
\hline $\begin{array}{l}\text { CAT } \\
\text { score, } \\
\text { n (\%) }\end{array}$ & $\begin{array}{l}\text { Count } \\
<10 \\
10-15 \\
>15\end{array}$ & $\begin{array}{l}186 \\
15(8.1 \%) \\
32(17.2 \%) \\
139(74.7 \%)\end{array}$ & $\begin{array}{l}190 \\
38(20.0 \%) \\
41(21.6 \%) \\
111(58.4 \%)\end{array}$ & .0008 \\
\hline $\begin{array}{l}\text { EQ-5D- } \\
3 \text { L utility } \\
\text { values }\end{array}$ & $\begin{array}{l}\text { Count } \\
\text { Mean }( \pm S D)\end{array}$ & $\begin{array}{l}183 \\
0.54( \pm 0.35)\end{array}$ & $\begin{array}{l}194 \\
0.64( \pm 0.30)\end{array}$ & $<0.0001$ \\
\hline $\begin{array}{l}\text { EQ-VAS } \\
\text { score }\end{array}$ & $\begin{array}{l}\text { Count } \\
\text { Mean }( \pm S D)\end{array}$ & $\begin{array}{l}190 \\
63.8( \pm 16.5)\end{array}$ & $\begin{array}{l}194 \\
65.0( \pm \mid 8.2)\end{array}$ & 0.4623 \\
\hline
\end{tabular}

Notes: Analysis of the relationship between the impact of COPD on health status, measured via the CAT; or quality of life (measured using the EQ-5D-3L) and represented by both the EQ-5D-3L utility value and the EQ-VAS score; and adherence to treatment, measured using the MMAS- 8 . The $p$-values were calculated using the Kruskal-Wallis test for the continuous variables (EQ-5D-3L and EQ-VAS) and the $\chi^{2}$ for the categorical variables (CAT score categories).

Abbreviations: CAT, COPD Assessment Test; EQ-5D-3L, 3-level EuroQol FiveDimension questionnaire; EQ-VAS, EuroQol Visual Analog Scale; MMAS-8, 8-item Modified Morisky Medication Adherence Scale. 


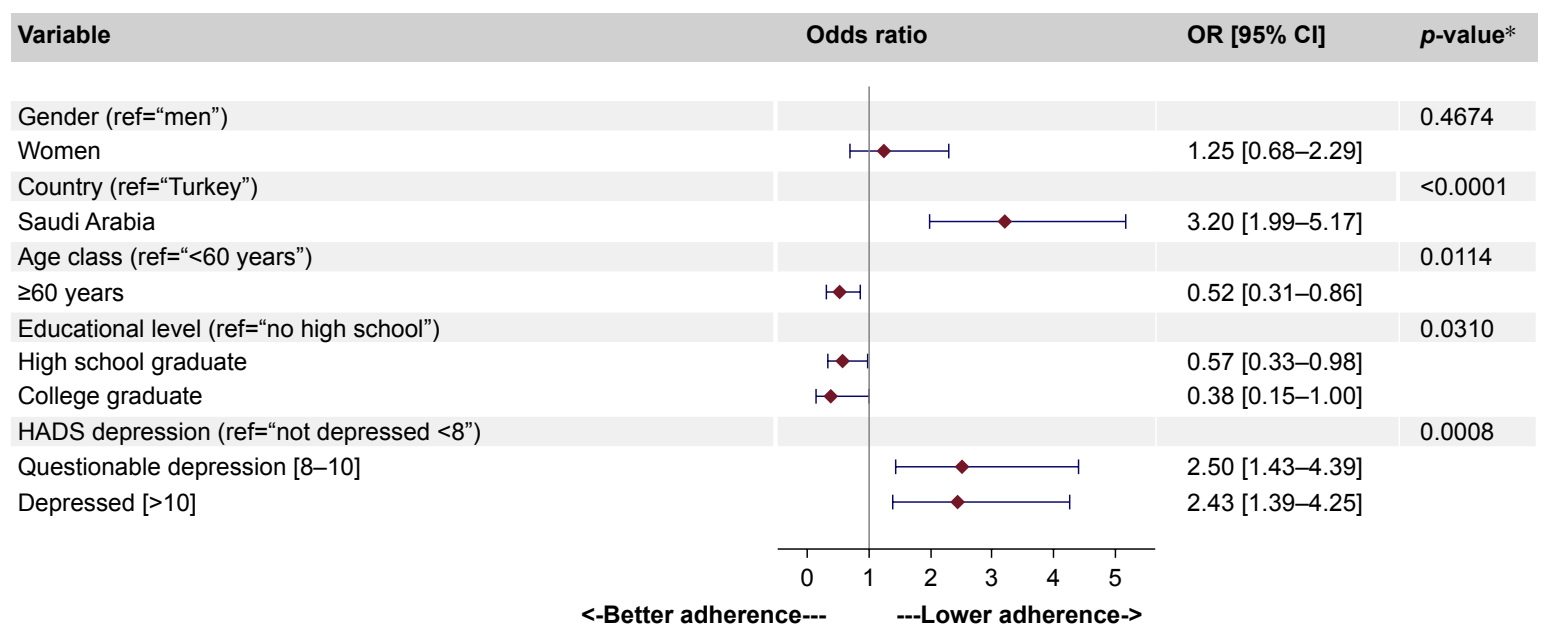

Figure I Multivariate analysis: predictors of non-adherence to COPD treatment.

Notes: Multivariate regression analysis on 355 patients: 170 with low (MMAS- $8<6$ ) adherence to treatment and I85 with medium or high (MMAS-8 $\geq 6$ ) adherence to treatment (low adherence versus medium/high adherence). ${ }^{*} p$-value of the Wald Chi-Square test from the logistic regression model.

Abbreviations: HADS, Hospital Anxiety and Depression Scale; MMAS-8, 8-item Morisky Medication Adherence Scale.

of age was also associated with better treatment adherence (OR 0.52) compared to $<60$ years of age $(p=0.0114)$. These results are presented in Figure 1.

\section{Impact of adherence to treatment on symptom severity}

After adjusting for age, gender, and country, a significant association ( $p=0.0314$ ) between patients who were adherent to treatment (defined as an MMAS- 8 score of $\geq 6$ ) and decreased disease impact (defined as CAT $\leq 15$ ) was observed (OR 0.56). These results are presented in Figure 2.

\section{Discussion}

This study marks the first time that COPD treatment adherence under real-world care conditions has been measured in Turkey and Saudi Arabia using a standardized questionnaire across multiple sites. The results demonstrate an association between poor treatment adherence, increased disease

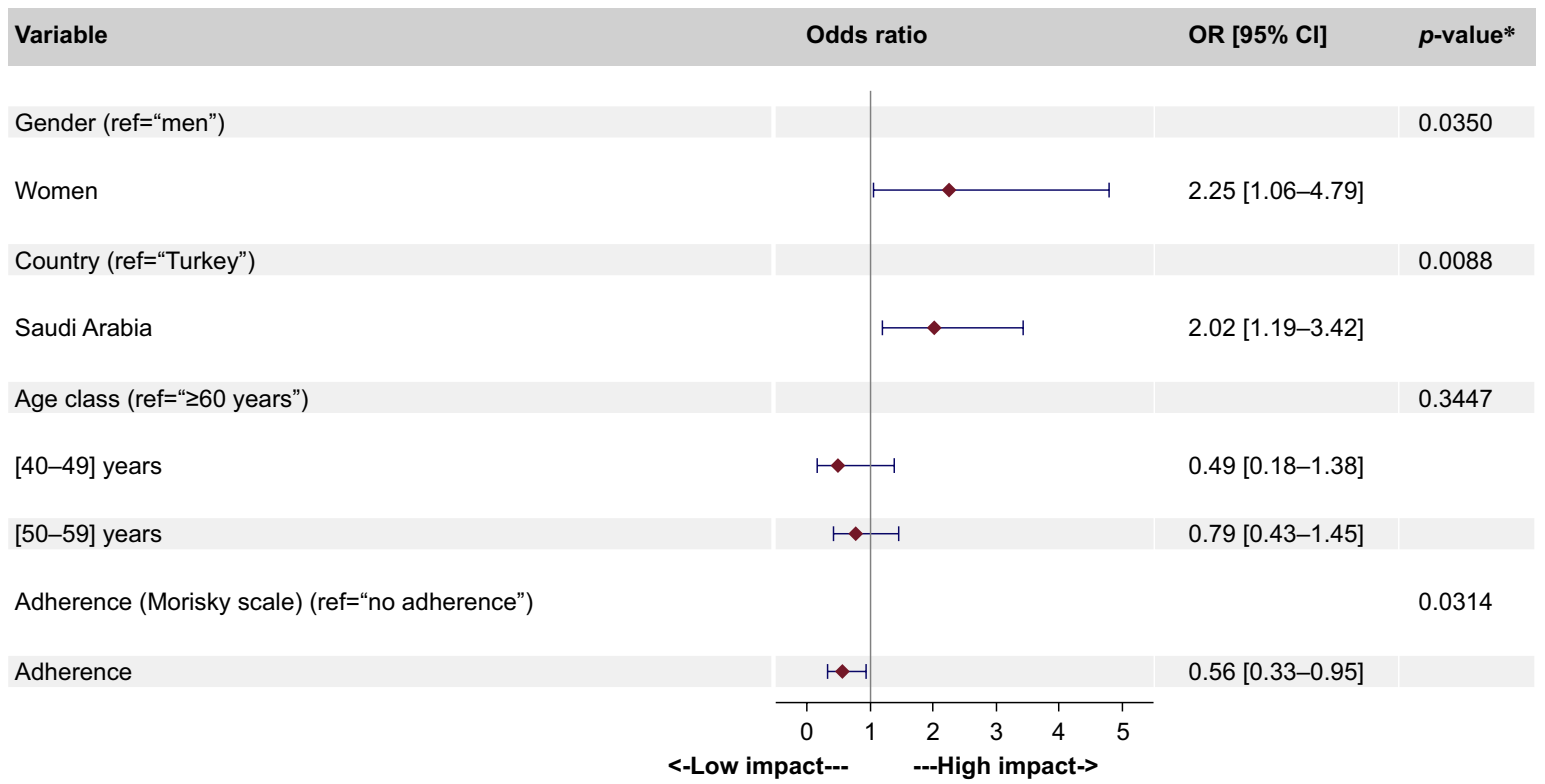

Figure 2 Association between treatment adherence and CAT score.

Notes: Adjusted analysis (by country, age, and gender) of the association between adherence to treatment and the impact of COPD on health status, measured by the CAT: analysis on 307 patients: 207 with a CAT score of $>15$ and 100 with a CAT score of $\leq 15$ (>I5 versus $\leq 15)$. *p-value of the Wald Chi-Square test from the logistic regression model. Abbreviation: CAT, COPD Assessment Test. 
impact, and reduced quality of life, and show a correlation between depression and lower treatment adherence and between older age and higher education with better treatment adherence. It should be noted that the observation of an association between adherence to treatment, depression, quality of life, and symptom severity makes no assumptions about causality.

Analysis of the demographics of the study population revealed the majority of COPD patients to be male and either current or former smokers. Smoking is considered to be a major risk factor for $\mathrm{COPD}^{19}$ and smoking rates in the Middle East and North Africa (MENA) are known to be high. Data from the BREATHE study reported as many as $39.5 \%$ of respondents in Turkey and $27.9 \%$ of respondents in Saudi Arabia were smokers. ${ }^{20}$ In addition, smoking rates in men still far exceed those in women in the MENA region, which may be a factor influencing the higher proportion of men in the study population. Data from the Global Adult Tobacco Survey Atlas published in 2015 reports the current smoking prevalence in Turkey in adults over the age of 15 as $48 \%$ in men and only $15 \%$ in women. ${ }^{21}$ This gender gap was also illustrated in the BREATHE study, which reported that, in Saudi Arabia, 38.7\% of men were smokers compared with only $7.4 \%$ of women, and in Turkey, $61 \%$ of men were smokers compared with only $23.5 \%$ of women. ${ }^{20}$ Interestingly in this study, $\sim 30 \%$ of patients in Saudi Arabia stated they were non-smokers. Since smoking is recognized as a major risk factor for COPD, this percentage is high. However, reporting of smoking in Saudi Arabia may be more difficult culturally than in Turkey, especially among women, who represent $25 \%$ of the ADCARE study population in Saudi Arabia, and those patients who reported as non-smokers may have been exposed to passive smoking. In addition, smoking is not the only risk factor for COPD; exposure to biomass fuels and smoke from wood burning has also been associated with COPD, ${ }^{22}$ which is common in Saudi Arabia. Indoor exposure to smoke from open wood fires or burning of biomass fuels has previously been found to be a risk factor for COPD among Saudi women. ${ }^{23} \mathrm{COPD}$ has also been independently associated with multiple conditions such as coronary heart disease ${ }^{24}$ and lung cancer. ${ }^{25}$ Therefore, it is not surprising that given the age of the subjects and the high prevalence of smoking, which is itself known to be a risk factor for many of these diseases, ${ }^{26}$ the majority of the study population reported at least 1 comorbidity.

Recent studies in chronic diseases ${ }^{4}$ and COPD specifically ${ }^{7}$ report poor levels of medication adherence. In line with this, approximately half (49.2\%) of COPD patients in this study reported low treatment adherence. Adherence was measured using the MMAS-8 - a scale that has been used widely to investigate adherence to medication in many disease areas including asthma ${ }^{27-29}$ and COPD, ${ }^{30-32}$ where it has been used to investigate adherence to inhaled medications.

In Turkey, there are very few studies investigating COPD treatment adherence, and most of those that have been published are single-center studies ${ }^{8-10}$ and focus on parameters such as inhaler technique. ${ }^{33}$ One Turkish study carried out in a single center, with a sample size of 59 patients, was designed principally to validate the Turkish version of the MMAS-8 questionnaire and reported that $46.4 \%$ of COPD patients had low adherence to treatment, ${ }^{9}$ which is comparable to the findings presented here, where $34.7 \%$ of COPD patients reported low adherence. Further research is needed to gain a better understanding of the levels of treatment adherence in COPD patients in these 2 countries, and repeating this study at different time points in the future would provide insights into this evolving problem.

Analysis of the results presented here shows that patients living in Saudi Arabia are more likely to be non-adherent to treatment than those in Turkey. The Ministry of Health $(\mathrm{MOH})$ in Turkey has prioritized chronic respiratory diseases in their national health programs. The National Control Program and Action Plan in Chronic Airway Diseases was put in place in 2009 through partnership with the World Health Organization Global Alliance against Respiratory Disease, with COPD being of special importance. ${ }^{34}$ Data from the 2015 Global of Burden of Disease study report COPD as the seventh leading cause of years of life lost in Turkey. In contrast, in Saudi Arabia, COPD did not feature in the top 10 diseases. ${ }^{1}$ The initiative from the $\mathrm{MOH}$ in Turkey to promote the awareness of COPD in the country and reinforce the treatment guidelines could be a contributing factor to the higher proportion of patients who are adherent to treatment in this country. Access to health care can influence a patient's adherence to treatment. There are a high proportion of expatriates living and working in Saudi Arabia, and the sociodemographics of this population may vary from the local population in terms of income, educational level, and access to health care. A breakdown of the local versus expatriate populations was not collected as part of the study. However, data collected on the educational level and health system coverage of the study population indicate that, in this study, access to health care is not an issue since the proportion of patients in both countries having no health system coverage is extremely low.

COPD is known to be a severely debilitating disease and has a substantially negative impact on the quality of life, ${ }^{35}$ and the extent of this impact has been linked to the 
severity of the disease as measured by the number or severity of exacerbations, ${ }^{36,37}$ and lung function, ${ }^{38}$ and the CAT score. ${ }^{39}$ In line with this, the patients enrolled in this study experience a high impact of COPD on their health-related quality of life as demonstrated by the low EQ-5D-3L utility values and EQ-VAS scores reported.

The findings from the ADCARE study also demonstrate that patients with poor adherence to treatment have a significantly lower mean EQ-5D-3L utility value than those with medium or high adherence, indicating a correlation between adherence to treatment and quality of life. Previously published studies provide inconsistent insight into this relationship. Some studies suggest that better quality of life leads to low adherence to treatment, as a result of the patient's perception of the benefits versus risk of taking their medication. ${ }^{40}$ Other studies suggest that, in line with the findings presented here, non-adherence to the prescribed treatment regimen is correlated with a negative impact on quality of life. ${ }^{41}$ Further research is needed to understand this complex relationship.

The published literature exploring a potential correlation between COPD symptom severity and adherence to treatment is complex, and comparison of data between the studies is difficult since different methodologies were used and different outcomes were measured. Data from the TORCH study reports no association between adherence to treatment and symptom severity as defined by Global Initiative for Obstructive Lung Disease (GOLD) stage, although patients with poor adherence did have lower baseline $\mathrm{FEV}_{1}$ and increased dyspnea. ${ }^{42}$ Similar findings were reported by another study investigating long-term adherence to home nebulizer use. ${ }^{43}$ Other studies report no significant association between adherence and severity, measured by $\mathrm{FEV}_{1} \cdot{ }^{40}$ Interestingly, a recent study based on the Continuing to Confront COPD International Patient Survey data reports an association between low medication adherence (measured using the MMAS-8) and increased symptom severity (measured by CAT). ${ }^{30}$ This is in line with the ADCARE data, which demonstrates that, overall, there is a greater proportion of patients reporting high disease impact (as measured by a CAT score of $>15$ ) in the low adherence group compared with those with medium or high adherence, an association that was confirmed in the multivariate regression analysis that showed that adherence to treatment was correlated with lower disease impact.

In this study, only $8.5 \%$ of patients had $\mathrm{FEV}_{1}<30 \%$ and only $15.5 \%$ of patients had FVC $<50 \%$, and yet nearly $50 \%$ used oxygen therapy in the previous 6 months. This suggests that the use of oxygen therapy was for breathlessness rather than as a long-term treatment for severe disease. Interestingly, despite the small proportion of patients with highly impaired lung function, two-thirds of patients reported a high impact of COPD on health status (CAT $>15)$. This is not an unusual finding, lower $\mathrm{FEV}_{1}$ has been shown to be associated with worse health, but the correlation is low, implying that some patients may have very poor health despite mild impairment in lung function. ${ }^{44} \mathrm{CAT}$ scores can show significant impairment in health status across all COPD severities, even in patients with mild disease, regardless of whether severity was assessed by a physician or classified by GOLD stage. ${ }^{45}$

Adherence to treatment for chronic diseases is a complex area. There are likely to be many factors involved that will vary depending on the patient and disease profile. Depression is known to be an important comorbidity of COPD, ${ }^{48}$ which is often untreated and is associated with poorer health outcomes. ${ }^{49}$ An association between depression and poor treatment adherence in chronic disease, ${ }^{50}$ and specifically in respiratory diseases such as asthma ${ }^{51}$ and COPD, ${ }^{52}$ has been reported in the literature. One such study was carried out in 78 patients in Turkey and showed that the presence of depressive symptoms was associated with low adherence to treatment. ${ }^{47}$ These findings are in line with the data presented here describing a significant correlation between depression and non-adherence to treatment.

Overall, both higher education and older age were associated with better adherence to treatment in the ADCARE study, but further research is needed to improve the understanding of the role that these factors play in treatment adherence for COPD. There is conflicting data published on the impact of demographic variables such as age and educational level on treatment adherence. Some studies have found that subjects who are older and better educated are more likely to be adherent to treatment. ${ }^{43,53}$ Other studies have shown no significant association. ${ }^{46}$ Some studies suggest that older age is a risk factor for non-adherence due to factors such as cognitive impairment, particularly forgetfulness and polypharmacy. ${ }^{54} \mathrm{~A}$ recent study carried out in Saudi Arabia, based on the findings from a National household survey, reported that older age was associated with better treatment adherence in subjects with chronic diseases. ${ }^{5}$ It has also been reported that patients with poor adherence often have insufficient understanding of their disease and its management options, and tend to rely more on natural remedies, ${ }^{55}$ which could be linked to patient education. In line with this, a recent study carried out among Palestinian geriatrics living with chronic disease reported a correlation between a higher level of knowledge about the medication and improved adherence to the treatment. ${ }^{56}$

ADCARE is one of the few studies addressing adherence to treatment in a real-life setting in the region, and the 
use of an identical study methodology in Turkey and Saudi Arabia enables pertinent comparisons to be made between the 2 countries. In addition, data on the impact of COPD on health status, quality of life, and adherence to treatment were collected using validated questionnaires (CAT, HADS, EQ-5D, MRC, and MMAS-8), which have been used widely, enabling comparison with data collected elsewhere.

Some limitations of the study should be noted. Since the study was conducted under real-world care conditions, patients visiting their physicians more often (and therefore it could be hypothesized that these patients have more severe disease) have a higher probability of being included in the study compared to those rarely seen. This may be a limitation in the generalizability of the study, although it has been shown in the multivariate analysis that the number of exacerbations (which is an indication of severity) is not associated with adherence. In addition, as is the case for all studies that require participants to recall data, there is a risk of recall bias and inaccuracy in the data collected. Furthermore, detecting poor adherence is challenging since there are many available methods to collect the data. In this study, patient self-reporting is used (the MMAS-8 questionnaire), which is the most cost-effective method but may result in an overestimate of adherence since patients frequently overreport medication adherence when completing such questionnaires. It is also noteworthy that the UK value sets were used to derive the utility values in the absence of country-specific reference values for Turkey and Saudi Arabia, on the advice of the EuroQol Group. Deriving the utility values based on country-specific value sets would be an important topic for future research.

In conclusion, the results presented here reveal that, in both Turkey and Saudi Arabia, adherence to COPD treatment is low and provides a benchmark for future studies investigating treatment adherence in these countries. The association between depression and poor adherence indicates that physicians should take into account psychological symptoms when treating COPD and consider counseling for those who are suffering. Another area for improvement is patient education, and educational level should be taken into account when treating patients with COPD to ensure patients understand the correct inhaler technique and benefits of regularly taking their medication. In addition, an association between non-adherence to treatment and higher disease impact, as well as a greater impact on the quality of life is demonstrated. Symptom management is a key aim of health care providers and policy makers, and therefore, improving adherence to treatment should be a key priority for decision-makers in order to reduce the burden of disease attributed to COPD.

\section{Data availability}

Editors can seek information from the corresponding author regarding whether anonymized patient level data can be made available.

\section{Acknowledgments}

Editorial support was provided by Sarah Osei-Ntem, an employee of GSK, in the form of manuscript development, collating of author comments, copy editing and referencing. Data analysis support was provided by Leyla Depret-Bixio, a former employee of GSK. The authors also express their appreciation for the contributions of Ahmed Abu Al Faraj, Caglar Karakurum, Selda Özdemir, Candice Pinto, Indira Umareddy (formerly employed at GSK), Tamer Elfishawy, Raef Gouhar, Saeed Noibi, Shireen Quli Khan, Nauman Rashid, Yalcin Seyhun (GSK), Aaicha Lahlou, Sophie Abadie, Salaheddine El Khadiri, Maria José Lopez (MS Health), and the participating CROs (Clinart and ZeinCRO) in different stages of the ADCARE study. The MMAS (8-item) content, names, and trademarks are protected by the US copyright and trademark laws. Permission for use of the scale and its coding is required. A license agreement is available from Donald $\mathrm{E}$ Morisky, ScD, ScM, MSPH, 14725 NE 20th St Bellevue, WA 98007, USA; dmorisky@gmail.com. Permission to use the EQ-5D-3L was provided by the EuroQol Research Foundation (C) EuroQol Research Foundation. EQ-5D ${ }^{\mathrm{TM}}$ is a trade mark of the EuroQol Research Foundation).

Funding for this study was provided by GSK (GSK study number 200368, GSK study acronym: ADCARE).

\section{Disclosure}

AEH, FA, and LT are employees of and shareholders in GlaxoSmithKline (GSK), which funded the ADCARE study. AD is a director of Foxymed, a medical communication and consultancy company, which participated in the design of the study and the interpretation of the results on behalf of GSK. The other authors report no conflicts of interest in this work.

\section{References}

1. Global, regional, and national life expectancy, all-cause mortality, and cause-specific mortality for 249 causes of death, 1980-2015: a systematic analysis for the Global Burden of Disease Study 2015. Lancet. 2016; 388(10053):1459-1544.

2. Mathers CD, Loncar D. Projections of global mortality and burden of disease from 2002 to 2030. PLoS Med. 2006;3(11):e442. 
3. Global, regional, and national disability-adjusted life-years (DALYs) for 315 diseases and injuries and healthy life expectancy (HALE), 1990-2015: a systematic analysis for the Global Burden of Disease Study 2015. Lancet. 2016;388(10053):1603-1658.

4. Napolitano F, Napolitano P, Angelillo IF. Medication adherence among patients with chronic conditions in Italy. Eur J Public Health. 2016; 26(1):48-52.

5. Moradi-Lakeh M, El Bcheraoui C, Daoud F, et al. Medication use for chronic health conditions among adults in Saudi Arabia: findings from a national household survey. Pharmacoepidemiol Drug Saf. 2016; 25(1):73-81.

6. Ingebrigtsen TS, Marott JL, Nordestgaard BG, et al. Low use and adherence to maintenance medication in chronic obstructive pulmonary disease in the general population. J Gen Intern Med. 2015;30(1):51-59.

7. Krauskopf K, Federman AD, Kale MS, et al. Chronic obstructive pulmonary disease illness and medication beliefs are associated with medication adherence. COPD. 2015;12(2):151-164.

8. Gulbay BE, Dogan R, Yildiz OA, et al. Patients adherence to treatment and knowledge about chronic obstructive pulmonary disease. Saudi Med J. 2006;27(9):1427-1429.

9. Oguzulgen IK, Kokturk N, Isikdogan Z. Turkish validation study of Morisky 8-item medication adherence questionnaire (MMAS-8) in patients with asthma and chronic obstructive pulmonary disease. Tuberk Toraks. 2014;62(2):101-107.

10. Ozyilmaz E, Kokturk N, Teksut G, Tatlicioglu T. Unsuspected risk factors of frequent exacerbations requiring hospital admission in chronic obstructive pulmonary disease. Int J Clin Pract. 2013;67(7): 691-697.

11. Jones PW, Harding G, Berry P, Wiklund I, Chen WH, Kline Leidy N. Development and first validation of the COPD assessment test. Eur Respir J. 2009;34(3):648-654.

12. Bestall JC, Paul EA, Garrod R, Garnham R, Jones PW, Wedzicha JA. Usefulness of the Medical Research Council (MRC) dyspnoea scale as a measure of disability in patients with chronic obstructive pulmonary disease. Thorax. 1999;54(7):581-586.

13. EuroQol-a new facility for the measurement of health-related quality of life. Health Policy. 1990;16(3):199-208.

14. Zigmond AS, Snaith RP. The hospital anxiety and depression scale. Acta Psychiatr Scand. 1983;67(6):361-370.

15. Morisky DE, DiMatteo MR. Improving the measurement of selfreported medication nonadherence: response to authors. J Clin Epidemiol. 2011;64(3):255-257; discussion 258-263.

16. Krousel-Wood M, Islam T, Webber LS, Re RN, Morisky DE, Muntner P New medication adherence scale versus pharmacy fill rates in seniors with hypertension. Am J Manag Care. 2009;15(1):59-66.

17. Morisky DE, Ang A, Krousel-Wood M, Ward HJ. Predictive validity of a medication adherence measure in an outpatient setting. J Clin Hypertens (Greenwich). 2008;10(5):348-354.

18. Min SY, Park DW, Yun SC, et al. Major predictors of long-term clinical outcomes after coronary revascularization in patients with unprotected left main coronary disease: analysis from the MAIN-COMPARE study. Circ Cardiovas Interv. 2010;3(2):127-133.

19. Eisner MD, Anthonisen N, Coultas D, et al. An official American Thoracic Society public policy statement: novel risk factors and the global burden of chronic obstructive pulmonary disease. Am J Respir Crit Care Med. 2010;182(5):693-718.

20. Khattab A, Javaid A, Iraqi G, et al. Smoking habits in the Middle East and North Africa: results of the BREATHE study. Respir Med. 2012; 106(Suppl 2):S16-S24.

21. Asma S, Mackay J, Song SY, Zhao L, Morton J, Palipudi KM, et al. The GATS Atlas. 2015 C. CDC Foundation, Atlanta, GA

22. Kurmi OP, Semple S, Simkhada P, Smith WC, Ayres JG. COPD and chronic bronchitis risk of indoor air pollution from solid fuel: a systematic review and meta-analysis. Thorax. 2010;65(3):221-228.

23. Dossing M, Khan J, al-Rabiah F. Risk factors for chronic obstructive lung disease in Saudi Arabia. Respir Med. 1994;88(7):519-522.
24. Sin DD, Man SF. Chronic obstructive pulmonary disease as a risk factor for cardiovascular morbidity and mortality. Proc Am Thorac Soc. 2005;2(1):8-11.

25. Kiri VA, Soriano J, Visick G, Fabbri L. Recent trends in lung cancer and its association with COPD: an analysis using the UK GP Research Database. Prim Care Respir J. 2010;19(1):57-61.

26. National Center for Chronic Disease Prevention and Health Promotion (US) Office on Smoking and Health. Reports of the Surgeon General. The Health Consequences of Smoking-50 Years of Progress: A Report of the Surgeon General. Atlanta, GA: Centers for Disease Control and Prevention (US); 2014.

27. Chiu KC, Boonsawat W, Cho SH, et al. Patients' beliefs and behaviors related to treatment adherence in patients with asthma requiring maintenance treatment in Asia. J Asthma. 2014;51(6):652-659.

28. Guenette L, Breton MC, Gregoire JP, et al. Effectiveness of an asthma integrated care program on asthma control and adherence to inhaled corticosteroids. J Asthma. 2015;52(6):638-645.

29. Ding B, Small M. Disease burden of mild asthma: findings from a cross-sectional real-world survey. Adv Ther. 2017;34(5):1109-1127.

30. Mullerova H, Landis SH, Aisanov Z, et al. Health behaviors and their correlates among participants in the Continuing to Confront COPD International Patient Survey. Int J Chronic Obstruct Pulmon Dis. 2016;11: 881-890.

31. Khdour MR, Kidney JC, Smyth BM, McElnay JC. Clinical pharmacyled disease and medicine management programme for patients with COPD. Br J Clin Pharmacol. 2009;68(4):588-598.

32. Baker CL, Gupta S, Goren A, Willke RJ. Adherence and satisfaction with oral versus other treatments among patients with chronic obstructive pulmonary disease (copd) in the u.s. 2012 national health and wellness survey. Value Health. 2013;16(3):A236-A237.

33. Aydemir Y. Assessment of the factors affecting the failure to use inhaler devices before and after training. Respir Med. 2015;109(4):451-458.

34. Yorgancioglu A, Turktas H, Kalayci O, et al. The WHO global alliance against chronic respiratory diseases in Turkey (GARD Turkey). Tuberk Toraks. 2009;57(4):439-452.

35. van Manen JG, Bindels PJ, Dekker FW, et al. The influence of COPD on health-related quality of life independent of the influence of comorbidity. J Clin Epidemiol. 2003;56(12):1177-1184.

36. Llor C, Molina J, Naberan K, Cots JM, Ros F, Miravitlles M. Exacerbations worsen the quality of life of chronic obstructive pulmonary disease patients in primary healthcare. Int J Clin Pract. 2008;62(4):585-592.

37. Solem CT, Sun SX, Sudharshan L, Macahilig C, Katyal M, Gao X. Exacerbation-related impairment of quality of life and work productivity in severe and very severe chronic obstructive pulmonary disease. Int $J$ Chronic Obstruct Pulmon Dis. 2013;8:641-652.

38. Stahl E, Lindberg A, Jansson SA, et al. Health-related quality of life is related to COPD disease severity. Health Qual Life Outcomes. 2005;3:56.

39. Marvel J, Yu TC, Wood R, Higgins VS, Make BJ. Health status of patients with chronic obstructive pulmonary disease by symptom level. Chronic Obstruct Pulm Dis. 2016;3(3):643-652.

40. Agh T, Inotai A, Meszaros A. Factors associated with medication adherence in patients with chronic obstructive pulmonary disease. Respiration. 2011;82(4):328-334.

41. Corden ZM, Bosley CM, Rees PJ, Cochrane GM. Home nebulized therapy for patients with COPD: patient compliance with treatment and its relation to quality of life. Chest. 1997;112(5):1278-1282.

42. Vestbo J, Anderson JA, Calverley PM, et al. Adherence to inhaled therapy, mortality and hospital admission in COPD. Thorax. 2009;64(11): 939-943.

43. Turner J, Wright E, Mendella L, Anthonisen N. Predictors of patient adherence to long-term home nebulizer therapy for COPD. The IPPB Study Group. Intermittent Positive Pressure Breathing. Chest. 1995;108(2): 394-400.

44. Jones PW. Health status measurement in chronic obstructive pulmonary disease. Thorax. 2001;56(11):880-887. 
45. Jones PW, Brusselle G, Dal Negro RW, et al. Properties of the COPD assessment test in a cross-sectional European study. Eur Respir J. 2011;38(1):29-35.

46. Khdour MR, Hawwa AF, Kidney JC, Smyth BM, McElnay JC. Potential risk factors for medication non-adherence in patients with chronic obstructive pulmonary disease (COPD). Eur J Clin Pharmacol. 2012; 68(10):1365-1373.

47. Turan O, Yemez B, Itil O. The effects of anxiety and depression symptoms on treatment adherence in COPD patients. Primary Health Care Res Dev. 2014;15(3):244-251.

48. Kunik ME, Roundy K, Veazey C, et al. Surprisingly high prevalence of anxiety and depression in chronic breathing disorders. Chest. 2005; 127(4):1205-1211.

49. Ng TP, Niti M, Tan WC, Cao Z, Ong KC, Eng P. Depressive symptoms and chronic obstructive pulmonary disease: effect on mortality, hospital readmission, symptom burden, functional status, and quality of life. Arch Intern Med. 2007;167(1):60-67.

50. DiMatteo MR, Lepper HS, Croghan TW. Depression is a risk factor for noncompliance with medical treatment: meta-analysis of the effects of anxiety and depression on patient adherence. Archi Intern Med. 2000;160(14):2101-2107.
51. Bender BG. Risk taking, depression, adherence, and symptom control in adolescents and young adults with asthma. Am J Respir Crit Care Med. 2006;173(9):953-957.

52. Albrecht JS, Park Y, Hur P, et al. Adherence to maintenance medications among older adults with chronic obstructive pulmonary disease. The role of depression. Ann Am Thorac Soc. 2016;13(9):1497-1504.

53. Rand CS, Nides M, Cowles MK, Wise RA, Connett J. Long-term metered-dose inhaler adherence in a clinical trial. The Lung Health Study Research Group. Am J Respir Crit Care Med. 1995;152(2):580-588.

54. Shrestha R, Pant A, Shakya Shrestha S, Shrestha B, Gurung RB, Karmacharya BM. A cross-sectional study of medication adherence pattern and factors affecting the adherence in chronic obstructive pulmonary disease. Kathmandu Univ Med J. 2015;13(49):64-70.

55. George J, Kong DC, Thoman R, Stewart K. Factors associated with medication nonadherence in patients with COPD. Chest. 2005;128(5): 3198-3204.

56. Najjar A, Amro Y, Kitaneh I, et al. Knowledge and adherence to medications among palestinian geriatrics living with chronic diseases in the West Bank and East Jerusalem. PLoS One. 2015;10(6):e0129240.

57. Brooks R. EuroQol: the current state of play. Health Policy. 1996;37(1):53-72.

\section{Publish your work in this journal}

The International Journal of COPD is an international, peer-reviewed journal of therapeutics and pharmacology focusing on concise rapid reporting of clinical studies and reviews in COPD. Special focus is given to the pathophysiological processes underlying the disease, intervention programs, patient focused education, and self management protocols.

\section{Dovepress}

This journal is indexed on PubMed Central, MedLine and CAS. The manuscript management system is completely online and includes a very quick and fair peer-review system, which is all easy to use. Visit $\mathrm{http}: / /$ www.dovepress.com/testimonials.php to read real quotes from published authors. 